\title{
Ensayo \\ Neurodiversidad, pedagogías de lo menor y singularidad subjetivizante: evaluar los aprendizajes a través de la perspectiva del desempeño en la acción
}

\author{
Fecha de recepción : 2020-04-29 • Fecha de aceptación:2020-08-19 • Fecha de publicación: 2020-09-10
}

\author{
Aldo Ocampo González \\ Centro de Estudios Latinoamericanos de Educación Inclusiva (CELEI), Chile \\ aldo.ocampo.gonzalez@gmail.com \\ https://orcid.org/0000-0002-6654-8269
}

\section{Resumen}

La construcción de instrumentos de evaluación y la implementación de experiencias de aprendizaje articuladas a través de los principios de la neurodiversidad asume un cambio significativo en la manera de concebir el aula y sus procesos, con el propósito de maximizar las oportunidades educativas del estudiantado acorde a su singularidad. El presente trabajo analiza las campanas de alarma y el aprendizaje escolar a través de sus dos síndromes más comunes: el de conocimiento frágil y el pensamiento pobre. Se exploran, además, las coordenadas que definen la perspectiva del desempeño en la acción y las cualidades de la comprensión, dispositivos claves en el diseño de instrumentos de evaluación. Posteriormente, el estudio se enfoca en la comprensión de la educación inclusiva y su relación con la justicia educativa, reconociendo que esta no sólo produce nuevos conocimientos, sino también poderosos ángulos de visión alterativos de la realidad, deviene en una concepción ontológica y pedagógica de lo menor, concebida en tanto compleja figuración imaginaria, política y epistémi$\mathrm{ca}$, una praxis y un lenguaje que diversas minorías construyen al interior de una forma pedagógica mayoritaria. 
Palabras clave: neurodiversidad, evaluación de los aprendizajes, tiempos múltiples heterogéneos, pedagogías de lo menor, inclusión.

\begin{abstract}
The construction of assessment instruments and the implementation of learning experiences articulated through the principles of neurodiversity assumes a significant change in the way of conceiving the classroom and its processes, with the purpose of maximizing the educational opportunities of the student body according to their singularity. This paper analyzes alarm bells and school learning through its two most common syndromes: fragile knowledge and poor thinking. In addition, the coordinates that define the perspective of performance in action and the qualities of understanding are explored, key devices in the design of evaluation instruments. Subsequently, the study focuses on the understanding of inclusive education and its relationship with educational justice, recognizing that this not only produces new knowledge, but also powerful alterative angles of vision of reality, it becomes an ontological and pedagogical conception of what minor, conceived as a complex imaginary, political and epistemic figuration, a praxis and a language that various minorities construct within a majority pedagogical way.
\end{abstract}

Keywords: neurodiversity, evaluation of learning, heterogeneous multiple times, pedagogies of the lesser, inclusion. 


\section{Introducción}

\section{El poder de la neurodiversidad y sus alcances para la práctica educativa}

La construcción de instrumentos de evaluación y la implementación de experiencias de aprendizaje articuladas, a través de los principios de la neurodiversidad, asume un cambio significativo en la manera de concebir el aula y sus procesos, con el propósito de maximizar las oportunidades educativas del estudiantado acorde a su singularidad. Entre las ideas más relevantes que nos permiten comprender el poder de la neurodiversidad en el aula, destacan: a) la concepción del espacio escolar en términos de hospitalidad y espacio b) la cristalización de entornos potenciadores que vayan más allá de espacios habitados por los significantes de la anormalidad -presencia de entornos menos restrictivos y activo -modificantes-, c) la fractura de los monocentrismos introducidos por la imposición del modelo epistémico y didáctico de educación especial que transfieren un ecosistema de esencialización y opresión al desarrollo de las prácticas educativas, reguladas mayoritariamente por el signo de la defectología, la filosofía basada en la carencia de autonomía propia, agudizando la acumulación del estigma. d) la neurodiversidad busca trabajar con los estudiantes en su plenitud -en este propósito coincide con los principios de la antroposofía en el estudio de la naturaleza humana-, e) se propone descubrir los talentos dones- de cada estudiante a lo largo del ciclo vital, f) su fuerza analítica ofrece una visión positiva y esperanzadora de las zonas no exploradas del ser.

El encuentro con la naturaleza humana y la singularidad no sólo persigue una re-lectura acerca del sujeto, las estructuras de escolarización y espacialidades, sino que adhiere a una idea mucho más profunda, la dislocación de la gramática escolar y de los intereses que nutren y vertebran los sistemas educativos, etc. Es un vector de separación de los contenidos escolares y las formas de nombrar cada una de las asignaturas, este busca una modificación a las formas de selección y legitimación de estos. La neurodiversidad es coherente con las formas de contractualismo social que abogan por las capacidades, coincide en este punto con la Teoría de Feuerstein -habita un doble vínculo-, es también una estrategia de recognición del sujeto. La neurodiversidad es otra forma de entender la gramática escolar, se encuentra profundamente interconectada con los principios de la Enseñanza para la Comprensión (EpC), específicamente al construir un currículo articulado sobre conexiones generativas que inviten a los estudiantes a ir más allá de sus posibilidades.

Un aula neurodiversa no es otra cosa que una espacialidad que trabaja a partir de las múltiples singularidades y tiempos heterogéneos de cada aprendiente. Tal como indica Armstrong (2012), "el aula neurodiversa cree en el desarrollo natural, orgánico, de cada individuo" (p.182). Es un trabajo que mantiene viva la imaginación, la novedad -neurotransmisor de la noradrenalina-, el juego, el movimiento consciente, la creatividad, la indagación, exploración, fantasía, todas ellas fuerzas básicas del aprendizaje que progresivamente son objeto de supresiones por las lógicas del capitalismo que regulan el sistema escolar en su conjunto. 
Nuestro cerebro es creativo, lúdico y caótico, así lo describe Jiménez (2000). El autor reafirma a su vez, que, desde su punto de vista, en la enseñanza:

no existe distancia, ni división de espacios: todo es infinito, todo es omnipresente, no localizado, no segregado. Esto explica que dos partículas que alguna vez vibraron juntas, seguirán respondiendo al movimiento una de la otra, a pesar de la distancia en el tiempo y en el espacio, como dos amantes que no pueden desenlazarse y olvidar el uno del otro (p.52).

Visto así, el espacio educativo adquiere la figuración de un holograma. Si la naturaleza compleja de nuestro cerebro opera en el caos, entonces la presencia de múltiples temporalidades heterogéneas es coherente, así como lo es la estructuración del aula desde una perspectiva de universo-mosaico y de lo menor, esto es, una espacialidad que altera sus dinámicas, ratifica que los mecanismos de disciplinamiento en la educación del cerebro necesitan de espacios de alta creatividad. Estas ideas demuestran que el cerebro aprende más y mejor por zonas de irregularidad, "el caos conduce a sistemas complejos" (Jiménez, 2000, p.10). En efecto, "lejos del equilibro, la materia tiene propiedades nuevas; igualmente, la variedad de los comportamientos posibles es muy sorprendente" (Prigogine, 1999, p.166).

El aprendizaje siempre acontece en una estructura disipativa, irruptiva y alterativa. Lo cierto es que, se aprende mejor en el movimiento consciente, en el desorden organizado y libre de error. Cada una de estas premisas, exige a su vez, la cristalización de un clima resonante, es la regularidad, la pasividad, la falta de imaginación intensiva y de desafíos lo que destruye la potencia cognitiva del ser. No existe aprendizaje sino hay placer, gusto y disfrute con aquello que experimentamos. La enseñanza en un aula neurodiversa enfrenta el reto de construir un poder subjetivo que fomenta la auto-organización cognitiva, contribuyendo a proponer nuevos modelos de acción.

\subsection{Las campanas de alarma y el aprendizaje escolar}

Las campanas de alarma son sinónimo de dos grandes síndromes descritos por Perkins (2003): síndrome del conocimiento frágil y síndrome del pensamiento pobre. Ambos pueden ser identificados en cualquier contexto de enseñanza, sus manifestaciones nos dan luces acerca de las debilidades de la comprensión y la fragilidad de las estructuras cognitivas. El primero está caracterizado por un efecto en el que "los estudiantes no recuerdan, no comprenden o no usan activamente gran parte de lo que supuestamente han aprendido" (Perkins, 2003, p.32). Mientras que, el segundo describe algo mucho más espinoso, "los estudiantes no saben pensar valiéndose de lo que saben" (Perkins, 2003, p.32). El pensamiento pobre describe situaciones de aprendizaje donde los estudiantes son incapaces de pensar por medio de lo que saben. En él, los aprendientes no son capaces de hacer inferencias, argumentar, escribir ensayos, solucionar problemas, saber cuándo aplicar un determinado concepto a la realidad, entre otras. Los estudiantes de pensamiento pobre utilizan la repetición -memorización- para retener el conocimiento en vez de utilizar técnicas más elaboradas o que estimulen los niveles superiores del pensamiento.

El conocimiento frágil se define como la pérdida o evaporación de los aprendizajes centrales o 
más relevantes que los estudiantes deben manejar para un determinado curso. Las dimensiones de esta singular manifestación de la cognición humana son: a) olvidado, caracterizado como una porción de conocimientos estudiados que han sido olvidados, o bien, desaparecen fácilmente. Este tipo de conocimiento denota ausencia de un funcionamiento activo ligado al proceso de pensamiento. b) Inerte, concebido como un conocimiento que se recuerda o no, en situaciones puntuales, la situación que mejor lo ejemplifica refiere a la preparación de exámenes para aprobar las materias, sin necesariamente perseguir un valor de cambio a partir del aprendizaje. Se encuentra íntimamente ligado al aprendizaje estratégico y superficial ${ }^{1}$. c) Ingenuo, tipología de riesgo cognitivo caracterizado por la mezcla e hibridación de teorías estereotipadas o concepciones erróneas sobre un determinado tema de estudio, en este nivel los estudiantes son capaces de entender el componente conceptual, sus dificultades se presentan al momento de explicar o interpretar algo, evidenciando concepciones erróneas casi intactas. Este conocimiento demuestra un nivel deficiente de comprensión (Perkins, 2003). d) Ritual, dimensión del aprendizaje que expresa una comprensión superficial y escasamente auténtica.

De acuerdo con Perkins (2003), la dimensión 'ritualista' refiere a un corpus de procedimientos a través de los cuales los estudiantes aprenden procedimientos necesarios para resolver determinados problemas. Este conocimiento demuestra un nivel deficiente de comprensión.

¿Qué es la enseñanza para la comprensión? El objetivo de la educación consiste en ayudarnos a hacer conscientemente aquello que no aprendemos de manera natural durante nuestra vida diaria. Por esto, la educación siempre debe preguntarse: ¿qué puede hacer para generar espacios accesibles al conocimiento a través de prácticas estimulantes? Para lograr dicho propósito sugiere Stone Wiske (2008) disponer de dispositivos de aprendizaje que les permitan transformar y desarrollar el conocimiento implícito inconsciente en conocimiento explícito consciente por medio de una participación activa que garantice un conocimiento generador a través de la retención, comprensión y uso activo del conocimiento.

Para Perkins (2006), la Enseñanza para la Comprensión (EpC) persigue que las personas construyan sus propios significados a partir de experiencias de aprendizaje, las cuales sean por lo general ricas en conexiones generativas. Aquí, un tema es generativo cuando ocupa un lugar "central" dentro de la materia o campo disciplinar, también cuando es "accesible" generando actividades de comprensión en docentes y estudiantes y, finalmente es "rico" cuando permite la capacidad de establecer conexiones entre diversos temas y materias. Como docentes debemos ser capaces de identificar dentro de cada una de nuestras asignaturas qué temas poseen estas características. La pedagogía de la comprensión invita a observar, analizar y reorganizar el currículum en torno a temas generadores que den origen y apoyo a diversas actividades mentales, ofreciendo a los estudiantes mayores oportunidades desde donde construir y aprender.

Enseñar para comprender y comprender para enseñar, supone no solamente transferir conocimientos sino crear posibilidades para su producción, ante la variedad de temas que abordan

1 El estudiante enfatiza sobre la intención de cumplir los requisitos de la tarea, tales como, memorizar la información necesaria para pruebas, exámenes o controles (procesuales y acumulativos). Es un enfoque basado en elementos sueltos, inconexos y sin integración. 
los programas de estudios, y en más, los requerimientos formativos derivados de las relaciones sociales de producción se vuelven necesario definir qué se debe enseñar y qué capacidades deben estimularse en nuestros estudiantes.

¿Qué significa comprender? según Blythe (1998), la comprensión se expresa al desarrollar la capacidad de hacer con un tema o contenido una variedad de cosas que estimulen el pensamiento, con la finalidad de aplicarlo de formas que vayan más allá del conocimiento y la repetición. La materialidad de la comprensión, en tanto umbral del aprendizaje, se manifiesta como un conocimiento activo disponible para el individuo, puede ser usado en diversas situaciones, un conocimiento que se recuerda siempre, promueve transferencia a nuevos contextos de desarrollo profesional: enfatiza sobre las capacidades de "liderar", "autogestionar" y "manejar conocimiento" y también se cristaliza mediante experiencias de aprendizaje que han sido estructuradas por el estudiante desde su propia experiencia, en contextos significativos y con crecientes momentos de reflexión que permitan utilizar lo aprendido de diversas maneras y en situaciones diametralmente diferentes.

Stone Wiske (2008), a diferencia de Perkins (2003), identifica cuatro niveles prototípicos de la comprensión, entre los que destacan: a) comprensión ingenua, captación de información directamente en el mundo, no evidencia conciencia de vínculos existentes entre la actividad y su aplicación en nuestra vida, b) comprensión de novatos, mecanismos y rituales c) comprensión de aprendiz, obedecen a modos de pensar disciplinarios y d) comprensión de maestría, críticos, holísticos, creativos y flexibles. Cada una de estas dimensiones describen la naturaleza multidimensional de la comprensión; no obstante, "la comprensión profunda entraña la capacidad de usar el conocimiento en todas las dimensiones" (Stone Wiske, 2008, p.239). Para comprender en profundidad el sentido y significado de los niveles de comprensión, es necesario referir a los desempeños de comprensión, es decir, diversas formas a través de las cuales, los sujetos demuestran lo aprendido. La Tabla 1 que se presenta a continuación, sintetiza las principales características descritas por Stone Wiske (2008). 
Tabla 1.

Estilos de comprensión

\begin{tabular}{|c|c|}
\hline $\begin{array}{l}\text { Dimensión de la comprensión } \\
\text { según Stone Wiske (2008) }\end{array}$ & Principales manifestaciones \\
\hline Comprensión ingenua & $\begin{array}{l}\text { Caracterizado por una desconexión de la vida real. } \\
\text { Carecen de formas reflexivas acerca de las que el conocimiento es expresado. }\end{array}$ \\
\hline Comprensión de novatos & $\begin{array}{l}\text { Describen los propósitos de la naturaleza del conocimiento. } \\
\text { Se vinculan a sistemas de mediación de las pruebas y los sistemas de } \\
\text { escolarización. } \\
\text { El desempeño de comprensión se basa en un mecanismo de aplicación, } \\
\text { contempla el desarrollo de un conjunto de pasos para lograr algo. }\end{array}$ \\
\hline Comprensión de aprendiz & $\begin{array}{l}\text { Reproducen modos de pensar disciplinarios. } \\
\text { Utilizan de forma flexible dichas ideas al interior de una determinada disciplina. } \\
\text { Según Stone Wiske (2008) "la construcción del conocimiento se ve como una } \\
\text { tarea compleja, que sigue procedimientos y criterios que son prototípicamente } \\
\text { usados por expertos en el dominio" (p.240). }\end{array}$ \\
\hline Comprensión de maestría & $\begin{array}{l}\text { Operan mediante integración: flexible y holística. } \\
\text { El estudiante se mueve con flexibilidad entre cada una de las comprensiones } \\
\text { antes indicadas. } \\
\text { Construcción del conocimiento complejo, resultado de complejas interacciones, } \\
\text { enfrentamientos, } \\
\text { Utilizan el conocimiento para actuar en la realidad, para intervenir en ella y } \\
\text { transformarla. } \\
\text { Fomentan una comprensión disciplinaria (meta-disciplinaria), el sujeto de } \\
\text { aprendizaje combina varias disciplinas para resolver un determinado problema. }\end{array}$ \\
\hline
\end{tabular}

Fuente: Stone Wiske (2008)

Las investigaciones de Perkins (2003 y 2006) y Stone Wiske (2008), sobre la visión representacional del conocimiento del estudiantado, señala que las representaciones mentales más efectivas que facilitan la comprensión se encontrarían determinadas por la utilización de estrategias o modelos de aprendizaje de tipo: analógico, construidos, depurados y concretos. La construcción del saber no depende exclusivamente de tales modelos, sino de la capacidad de indagación e innovación del docente en la creación de dispositivos de mediación didáctica.

- Modelo analógico: estrategia que permite desarrollar representaciones mentales en torno a algún tipo de analogía con el fenómeno real que interesa estudiar. En este modelo las estrategias buscan un propósito inmediato.

- Modelos construidos: corresponden a modelos de pensamiento analógico construidos con un propósito inmediato. Se basan en un conocimiento intuitivo y en una concepción errónea del saber.

- Modelo depurado: las estrategias de enseñanza deben intentar identificar de cada contenido, reduciendo al máximo los elementos que generen confusión en la explicación del docente.

- Modelo concreto: se presentan de manera concreta al fenómeno o contenido en estudio mediante la utilización de imágenes visuales, ejemplos, demostraciones experimentales, etc. Cada explicación es acompañada de la vivencia directa con el objeto de estudio involucrado. 
Aunque existan otras estrategias y modelos de aprendizaje que nos pueden ayudar a incrementar la comprensión, es de vital importancia desarrollar procedimientos didácticos y evaluativos que permitan a los educadores acercarnos al significado de las representaciones y a la arquitectura cognitiva del estudiantado.

Al desarrollar rutinas de pensamiento que estimulen la comprensión profunda y el pensamiento complejo de los estudiantes, será posible desarrollar diversas oportunidades para abandonar toda visión intuitiva y asistemática del pensamiento por una más constructiva, consciente y reflexiva del mismo; las que al utilizarse una y otra vez instarán a los estudiantes a estar atentos a los problemas y oportunidades que emerjan de cualquier desafío cognitivo subrayando, la importancia de solucionar flexiblemente los problemas epistémico-didácticos presentes en cada disciplina constitutiva del currículo escolar.

El propósito fundamental que persigue la Enseñanza para la Comprensión (EpC), consiste en el desarrollo de un modelo pedagógico vertebrado en torno al pensamiento, donde los estudiantes aprenden a reflexionar sobre lo que aprenden y comprenden. Frente a este desafío, es fundamental que los docentes enfrenten a sus estudiantes a experiencias de aprendizaje que les permitan retener, comprender y usar activamente el conocimiento mediante situaciones en las que los alumnos reflexionen sobre lo que están aprendiendo, cómo lo están aprendiendo y con lo que están aprendiendo.

Los alumnos aprenden a comprender mediante el desarrollo de acciones, estrategias y experiencias de aprendizaje, fundamentalmente reflexivas, es decir, prestando atención a los procesos implicados en la construcción del conocimiento, partiendo de sus fortalezas y superando sus debilidades.

Las estrategias de aprendizaje que deseen incrementar la comprensión deberán dedicar mayor parte de su tiempo a actividades que exijan tareas intelectualmente estimulantes, tales como explicar, generalizar, y, en última instancia, aplicar esa comprensión a sí mismos. Se debe hacer a través de un feedback constante durante todo el proceso de aprendizaje, a fin de poner en primer plano el compromiso reflexivo con los desempeños de comprensión sugeridos por el docente.

Los estudiantes aprenden más a fondo cuando "organicen los hechos, los relacionen con el conocimiento anterior, utilizan asociaciones visuales, se examina a sí mismo y elaboran y extrapolan lo que están leyendo o escuchando" (Perkins, 2003, p.40). Estos aprenden a comprender por medio de estrategias que les ayuden a procesar de forma más elaborada y que brinden prioridad al desempeño reflexivo en tareas complejas que admiten más de una respuesta. Por tanto, un buen aprendizaje es el resultado de un compromiso reflexivo del alumno con el contenido de la enseñanza.

\subsection{La perspectiva del desempeño en la acción: demostrar lo aprendido}

La Enseñanza para la Comprensión es un poderoso modelo que atiende la multiplicidad de singularidades presentes en el espacio escolar -en adelante de lo menor-. La perspectiva del desempeño en la acción es una idea que extraigo de este modelo, descrita como un conjunto 
de actividades que permiten pensar y actuar con flexibilidad. Justamente, la perspectiva del desempeño en la acción, acontece en la flexibilidad del pensamiento y en la capacidad de demostrar lo aprendido. La acción es lo que debemos aprender a evaluar, es aprender a capturar las diversas manifestaciones de la profundidad de lo aprendido. La perspectiva del desempeño en la acción consiste en desplegar diversos recursos y espacios para demostrar lo aprendido.

La comprensión se evalúa en la acción, en el eje mismo de producción del aprendizaje,

quiere decir ni más ni menos que ser capaz de desempeñarse flexiblemente en relación con el tópico -contenido-2: explicar, justificar, extrapolar, vincular y aplicar de manera que van más allá del conocimiento y la habilidad rutinaria. Comprender es ser capaz de pensar y actuar con flexibilidad a partir de lo que uno sabe (Stone Wiske, 2008, p.73).

En relación a los retos de la evaluación, la perspectiva del desempeño en la acción se orienta a una "visión que exige alcanzar una representación mental que capta lo que ha de componerse" (Stone Wiske, 2008, p.75).

La evaluación, en este contexto, debe ayudarnos a pensar en imágenes -capturar su esencia-, cada una de ellas forma un esquema de acción. La visión representacional de la comprensión es clave en el diseño de instrumentos de evaluación en el contexto de la educación de la mente y la neurodiversidad. Lo que evaluamos en este contexto son las estructuras de representación y la captura la comprensión en acto, aquello que hace el estudiante para dar cuenta de lo aprendido. Comprensión en acto y visión representacional del aprendizaje -imágenes mentalesse encuentran profundamente imbricadas, estas son dos de las principales características fundamentales a considerar en el diseño de la evaluación a través de la neurodiversidad.

\subsection{Evaluación de los aprendizajes y neurodiversidad: apostar por las cualidades de la}

\section{comprensión}

Sin duda, la evaluación es siempre una tarea espinosa para cualquier educador (Stone Wiske, 2008), especialmente en el contexto de una pedagogía neurodiversa, puesto que, el reto reside en la creación de dispositivos que permitan capturar las cualidades de cada aprendizaje y sus ramificaciones. En este apartado se exploran las formas de inter-penetración e imbricación de los términos y las funciones de la evaluación en el contexto de la neurodiversidad.

Otro enigma central en la escolaridad es el aprendizaje. Fundamentalmente sugiere un examen acerca de lo que realmente logra cada aprendiente. La evaluación de los aprendizajes en el contexto de la neurodiversidad centra su interés en el proceso mismo de comprensión, es decir, promueve el conocimiento y permite que el docente conozca qué se ha comprendido y, partir de ello, orienta los pasos de la enseñanza y del aprendizaje acorde a la singularidad de cada estructura cognitiva del estudiantado.

Este tipo de evaluación es entendida como una dimensión crítica-reflexiva del proceso de 
aprendizaje-enseñanza y no como un momento final de certificación. En efecto, "el proceso mediante el cual se lleva a cabo el seguimiento del progreso de los estudiantes dentro de un área, de manera que las decisiones puedan adoptarse del mejor modo posible con el fin de que facilite un mayor aprendizaje" (Klenowski, 2004, p.102). El propósito fundamental de este estilo de evaluación es describir las capacidades y los desempeños de comprensión de cada alumno, un punto clave consistirá en describir el corpus de descriptores de progreso que cada estudiante experimenta en función de las particulares demandas de cada experiencia de aprendizaje.

Los niveles de la comprensión identificados por Stone Wiske (2008), son: contenido, resolución de problemas, nivel epistémico y de investigación. Cada uno de estos niveles se encuentra íntimamente articulados con las cualidades multidimensionales del aprendizaje, y cada uno es clave en el diseño de instrumentos de evaluación.

Según Perkins (2003), la dimensión de contendido se refiere a datos e información de carácter instrumental y de transmisión de la información. La dimensión referida a resolución de problemas ofrece soluciones directas a los nudos críticos más comunes que enfrenta cada asignatura. Lo epistémico, por su parte, asume los hábitos mentales en términos de generalizaciones y explicaciones acerca de lo que hacen los estudiantes. Finalmente, en el nivel de investigación, los estudiantes han fortalecido sus aprendizajes y poseen la capacidad de construir nuevos conocimientos. De acuerdo a las tensiones de escolarización, es necesario fortalecer el nivel de contenido en las prácticas educativas, con el objeto de fomentar poderosas imágenes mentales.

Todo ello enfrenta el desafío de fortalecer los niveles superiores de los hábitos mentales y su desarrollo en cada una de las secciones del currículo escolar y formas de mediación didáctica y evaluativas. Además de configurar representaciones potentes, ¿qué cosas cultivan la comprensión en el espacio escolar?, ¿de qué manera las disciplinas apoyan dicho proceso?, ¿qué actividades fomentan la construcción de imágenes mentales poderosas a través de las disciplinas y su naturaleza multidimensional y multiestructural? El aprendizaje en el modelo de Enseñanza para la Comprensión es de carácter reflexivo, creando el conocimiento a través de la transformación de la experiencia. Reconoce la presencia de dos fuentes facilitadoras de la experiencia de aprendizaje: la "experiencia concreta y vivencial" y la "experiencia mental de conceptualización abstracta". Su trabajo se articula a favor de la creación de operaciones cognitivas permitiendo a los estudiantes ser capaces de producir sus propias ideas, creencias y conocimientos a partir de lo que están aprendiendo.

Para Tishman y Palmer (2005), la educación del pensamiento contribuye a:

- Consolidar el conocimiento: permite comprender al docente las relaciones que desarrollan los estudiantes al procesar la información (secuencia integrada de acciones y conexiones), 
facilitando un pensamiento auténtico en vez de solo memorizar hechos.

- Demostrar el valor de la colaboración intelectual: promueve el intercambio crítico y propositivo de las ideas, argumentos y concepciones sobre un objeto de estudio puntual, integrando sus ideas a la constelación de saberes de su propia clase. Este punto se vincula al aprendizaje colaborativo ya que ilustran un "entendimiento conceptual colaborativo sobre un tema que es más amplio y más complejo que cualquier concepción individual de un(a) estudiante" (p. 34).

- Una modificación en la cultura de la clase: supone la consecución concreta de un clima subjetivo en el aula, incrementa el "despliegue de las ideas y preguntas de los(as) estudiantes, las representaciones visibles de sus ideas en desarrollo e incluso del tono de la interacción en el aula envían el mensaje de que al pensamiento se le dan gran valor" (p. 37).

El desarrollo intelectual o el proceso de aprender a pensar profundamente se produce como resultado de la interacción dinámica de todos estos aspectos, agregándose los siguientes componentes: metacomponentes, refieren a la capacidad de los estudiantes para planificar y tomar decisiones al momento de resolver un problema, desempeño, corresponden a actividades donde se implementa el plan y la toma de decisiones concretamente, adquisición, corresponde a la fase de procesamiento, transferencia, describe las formas de aplicación de la información retenida desde un contexto a otro y la retención. Todo esto conduce al uso y procesamiento activo del conocimiento en un espiral creciente de aprendizaje y exploración en los tópicos de configuración del currículo escolar.

La organización del aprendizaje alrededor de temas que les generen gran interés y motivación, sugiere atender a metas de comprensión explícitas, ofreciendo muchas oportunidades para aplicar lo que los docentes están aprendiendo y, con ello, realizar una evaluación continua que permita una retroalimentación constructiva sobre las configuraciones de sus desempeños de aprendizaje.

La EpC se propone atacar dos errores persistentes en la enseñanza convencional: la imposibilidad que los estudiantes usen bien sus mentes a través del tipo de actividad que promueven, y la carencia de valor de los aprendizajes más allá de éxito requerido por las metas de carácter eficientistas impuestas por el sistema social. El desafío que enfrentan los docentes es componer climas de aprendizajes que permitan a los estudiantes usar sus mentes, procesos que son vertebrados en torno a los elementos sugeridos en la Figura 1 que se presenta a continuación. 


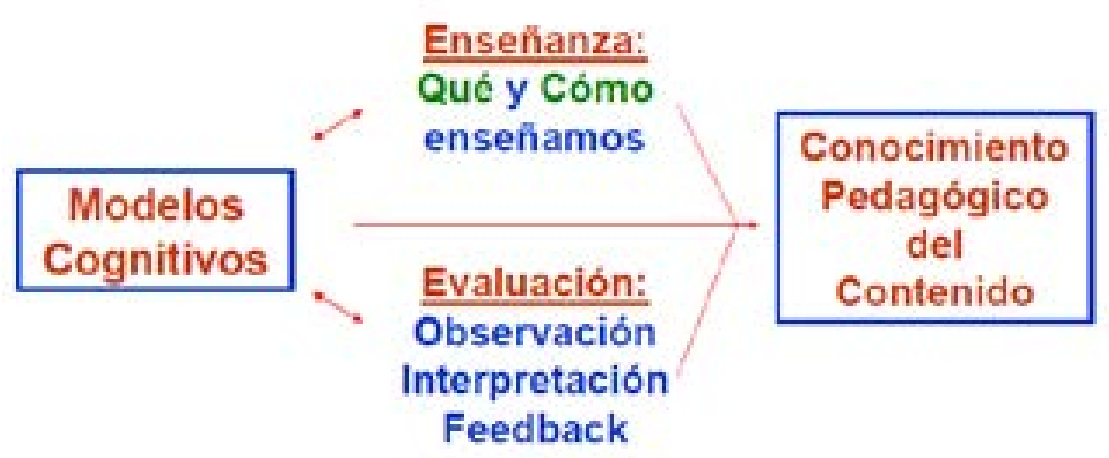

Figura 1. Diseño de experiencias de aprendizaje y formas de transitividad desde el conocimiento implícito al conocimiento explicito consciente.

Fuente: Talanquer (2010).

\section{Pedagogías de lo menor: una praxis de educativa para las singularidades múltiples}

El concepto pedagogías de lo menor fue propuesto por primera vez en el prólogo del libro Pedagogías Queer, publicado en 2018, por el fondo editorial del Centro de Estudios Latinoamericanos de Educación Inclusiva (CELEI). La sección del sintagma referida a 'lo menor' recupera los planteamientos de la revolución molecular introducida por Deleuze, inaugura una forma ontológica que versa sobre la potencia de la singularidad.

Pedagogías de lo menor no es sinónimo de una forma pedagógica de lo minoritario concebida desde un enfoque de asimilación de lo que supuestamente excede las estructuras de escolarización-, sino, es una compleja figuración imaginaria, política y epistémica, una praxis y un lenguaje que diversas minorías construyen al interior de una forma pedagógica mayoritaria -lo establecido-. Es signo de alteratividad de las estructuras de escolarización y sus lógicas de funcionamiento. Su lenguaje, su funcionamiento epistémico-político y metodológico se ve afectado por la fuerza de la des-territorialización y re-territorialización permanente. Es una forma pedagógica que se abre a otras modalidades de imaginación epistémicas, una figuración altamente creativa.

Una de las principales características de las pedagogías de lo menor es su marcado componente político que opera sobre un tejido conectivo de orden intersubjetivo, en el que cada una de sus múltiples singularidades se unen en zigzag configurando un espacio, algo fuera de serie, una singularidad membránica que transforma fronteras.

Pedagogías de lo menor es un espacio de transposición de lo individual-advertencia freudiana de producción de seres en masas, sujetos homogéneos, etc.- a lo singular -captura de lo inextricablemente humano, aquello que libera al ser de las ataduras de lo abyecto, de la ideología de la normalidad, del individualismo metodológico, de la neutralización y la cosificación de su identidad-. En este particular emerge el valor colectivo del propio concepto, resituando el universo conceptual de la educación inclusiva en el centro crítico de la multiplicidad, es decir, aquello que 
no se cierra fácilmente, que opera por redoblamiento, es un dispositivo de enunciación colectiva singularizante.

Este término es un poderoso tropo político-ético y un dispositivo de producción de subjetividades singularizantes, fomenta la emergencia de una concepción y praxis que deforma los modos canónicos de concebir las formas pedagógicas, es una zona de agitación y dispersión. Trabaja en la emergencia de otra gramática pedagógica, es un contra-espacio epistémico y político, una forma revolucionaria.

Esta concepción puede ser concebida en tanto singulares modos de subjetividad construidas en espacios hegemónicos por agentes de la revolución molecular, sus operatorias acontecen en el movimiento de des-territorialización y re-territorialización múltiple, es una estrategia de imbricación e inter-penetración de lo singular con lo político devenido en la construcción de un espacio alterativo fuera-de-serie. En tanto forma imaginativa y entendimiento complejo emerge como un ejercicio de dislocación instaurado dentro de un espacio mayoritario y hegemónico.

Pedagogías de lo menor entraña una compleja imbricación ontológica y política. Hablamos así, no sólo de una ontología de lo menor, sino también, de un espacio pedagógico y político. Es una fuerza que performa el tiempo, los espacios, las pasiones, las afectividades y los pensamientos. Es un vector altamente creativo. Esta modalidad pedagógica subvierte el problema ontológico, político y estético de la enseñanza y sus formas condicionales requeridas por la educación inclusiva.

La espacialidad pedagógica y política que construyen las pedagogías de lo menor -zona potentecreativa $^{3}$ - se encuentra en permanente devenir, es tallado por la fuerza del acontecimiento. Lo menor es una figuración de intensidad creativa y poderosa forma de intervención política. Es una unidad relacional que toma la forma de circunstancias y figuraciones de trascendencia abstracta. Tal como afirma Núñez (2010), es un movimiento de minorización que inaugura "un pliegue que altera nuestras sociedades y modos de pensar: los modos de vida. Es a través de la formulación de una ontología menor como sistema abierto, inmanente y unívoco, como Deleuze nos conduce inmediatamente a la ontología política y a la ontología estética" (p.47).

La política ontológica de la educación inclusiva rechaza cualquier forma de fijación de la esencia y de figuras predeterminadas como sucede a través de la imposición del modelo epistémico y didáctico de la educación especial devenida en la travestización de sus contornos teóricos y metodológicos, reafirmando una política discursivamente liberal, gobernada soberanamente por un corpus de esencialismos-individualismos que operan estratégicamente en la enunciación y escuchabilidad del campo. En contraposición ontológica, la concepción del ser legitimado a la luz de la autenticidad del dominio se encuentra estrechamente

apegada a las circunstancias, a las potencias y a las minorías (...) como es el pensar activo mismo. Y todo ello porque deviene estética, es decir, porque deja de encargarse de lo que está fuera de todo espacio y tiempo y se ve inmiscuida con el pensar y el alterar creativamente los espacios y los tiempos mismos (Núñez, 2010, p.48),

3 Corresponde a un peculiar modo de resistencia en el trabajo epistemológico de la educación inclusiva. 
La inclusión es una figuración creativa de lo nuevo que recompone la potencia del ser. La educación inclusiva establece un nuevo código ontológico abierto que emerge de la comprensión de las circunstancias contextuales, relacionales y micro-prácticas y no de las esencias, el sujeto o, mejor dicho, la multiplicidad de sujetos que emergen de ella, no son figuraciones previamente determinadas, es un ser en permanente invención. "Del mismo modo como "menor" ya nombra siempre, independientemente pero indisociablemente unida a la política, una potencia propia de la filosofía para crear modos de vida al margen de los poderes" (Núñez, 2010, p.48). ¿A qué movimiento ontológico nos conduce lo menor? A una trama de relaciones que activan la proliferación de singulares modos de subjetividad crítica y afectividades heterológicas.

\subsection{Construyendo un espacio educativo fuera-de-serie: lo heterotópico}

Los aportes de la Escuela de la Geografía Brasileña y de Massey (1994), resultan determinantes en la definición de la noción de espacio pedagógico de la inclusión, concebido en tanto unidad relacional siempre contingente y dinámica, un territorio pensado en términos de una frontería -espacio indeterminado-, "concebido como algo que se forja al margen del territorio tradicionalmente habitado, posibilitando la lucha continua, la conjugación de sus múltiples sistemas de razonamientos y elementos que definen su carácter performativo" (Ocampo, 2018, p.105).

Pensar desde coordenadas la espacialidad pedagógica de la educación inclusiva auténtica, supone la creación de un pensamiento, de un sistema argumental y de una práctica educativa fuera-de-serie, consolida un espacio no existente en la gestión de oportunidades educativas. Cada una de sus coordenadas de configuración desafían la composición dialéctica tradicional cuya analítica trabaja a favor de procesos de inclusión/exclusión reducidos a una mecánica binarista. Recuperando los planteamientos de Lazzarato (2006), es posible atender a las articulaciones de la gramática escolar en los sistemas occidental céntricos, tramas en las que:

el aparato general del imperio procede, en el primer lugar, a la inclusión de todos en el espacio imperial, de tal modo que el 'velo de ignorancia' respecto de las diferencias permite la sumisión jurídica de todos. En segundo lugar, las diferencias son afirmadas en tanto culturales (y no políticas), lo que permite la integración de los trabajadores como mecanismo eficiente de control del proceso de trabajo, de manera que el poder imperial se halla, pues, en la movilidad, la flexibilidad y la contingencia. Por último, el imperio procede a la administración de la inclusión y la diferencia (p.33).

La intelectualidad dominante sobre diferencia e inclusión es estructurada desde la vagancia epistémica, reafirmando de un sistema de ignorancia que ubica su función en torno a un corpus de esencialismos-individualismos, cuya materialidad del derecho a la educación reproduce un silencioso efecto universal y homogenizante. Al ser un campo y una expresión de la multitud, la inclusión actúa como un sistema de renovación del pensamiento y de las condiciones subjetivas y materialistas que afectan a los modos de practicar y experimentar la enseñanza y el aprendizaje.

Para Deleuze (1968), la 'multitud' es fuente de ilimitadas configuraciones, es la imagen de lo uno y lo múltiple, reafirmando el carácter dinámico de los principios educativos. ¿Cuáles son los 
conceptos que permiten hacer el anclaje ontológico, epistémico y metodológico en la construcción de un nuevo orden educativo coherente con estas características? Esta operación procederá

desde la perspectiva de Deleuze, oscila entre la afirmación productiva y diferencial de la materia viva que es su principio inmanente, -dando lugar a una forma singular que se vertebra en un proceso creativo-, y un proceso de estratificación que implica su sujeción al Juicio de Dios, es decir, la negación de su propia dimensión creativa en aras del enaltecimiento de la figura de lo Uno o lo Mismo (Deleuse, 1968, p. 145).

\subsection{Heterocronías: la noción de tiempo en la educación inclusiva}

La pregunta por la cuestión del tiempo en la educación inclusiva constituye otro punto análisis relevante en la investigación epistemológica impulsada por Ocampo (2020). Nuevamente, aquí nos enfrentamos a otro punto espinoso, si tuviese que elegir un concepto vertebrador de la argumentación, sin duda este recaería en el par analítico-político alterativo-alteratividad, cuyo significante se opone diametralmente a los proporcionados y legitimados por el concepto de alternativas. Trabaja a favor de aquello que cambia sustantivamente el orden de las cosas.

El análisis del tiempo pedagógico constata una articulación que entraña una fuerza performativa que altera el orden de la normalización y la homogenización de las coordenadas temporales implicadas en la enseñanza y en la experiencia educativa. La política de temporalización que asume la educación inclusiva, construida en base a la imposición del modelo epistémico y didáctico de la educación especial, refuerza entendimientos de orden monocrónicos -tiempo único-. En contraste, la noción de tiempo pedagógico coherente con las demandas de la multiplicidad de singularidades, refuerza una concepción de múltiples tiempos heterogéneos entrecruzados, ideas que son albergadas bajo la noción de heterocronías de aprendizaje.

Esta concepción del tiempo devela una operación que transpone aspectos medulares de regulación de la teoría educativa, como es el salto desde lo mono-crónico a lo heterocrónico sinónimo de un tiempo pedagógico múltiple-, es decir, el paso de un tiempo único, fijo, lineal, positivo, etc., a un tiempo gobernado por múltiples posibilidades, estriado, nómade, etc. Lo mencionado inaugura un nuevo signo pedagógico. La heterocronía es coherente con la heterogeneidad del espacio social y pedagógico, y, ontológicamente pertinente con la multiplicidad singularizante del sujeto.

Monocronía es sinónimo de cronosistema 4 -forma de ordenación específica-, mientras que, las heterocronía es sinónimo de espacio pedagógico de lo menor, abierto y en permanente devenir unidad relacional multi-temporalizado-. Es una forma de des-habitualización y de performatividad de las lógicas de trabajo escolar. Esta discusión no se cierra exclusivamente en la trama educativa, sino que afecta a todos los campos del desarrollo humano.

A través de las nociones de múltiples singularidades -dimensión crucial de la política ontológica de la inclusión y universo mosaico es posible reconocer la convivencia de múltiples tiempos en la

$4 \quad$ Concepto ampliamente abordado por Terigi (2004). En la acción pedagógica consiste en hacer todos lo mismo, al mismo ritmo y en el mismo tiempo. También es concebida como única cronología de aprendizaje. 
regulación de la trama escolar. Tal como señala Terigi (2004),

cuando hablamos de aprendizajes monocrónicos, nos referimos a que la organización escolar descansa en el supuesto de proponer una secuencia única de aprendizajes a cargo del mismo docente, sostener esta secuencia a lo largo del tiempo con el mismo grupo de alumnos, de modo tal que, al final de un proceso más o menos prolongado de enseñanza o período (por ejemplo, de un trimestre o de un ciclo lectivo) y desarrollada la enseñanza tal como ha sido prevista, todos los miembros del grupo hayan aprendido las mismas cosas (p.104).

La monocronía o el tiempo pedagógico por antonomasia reafirma el carácter mutilado de las prácticas de escolarización. Este propósito en el contexto epistemológico de la educación inclusiva, observa cómo los espacios escolares han operado a través del principio de identidad -pensamiento de lo idéntico o de lo mismo (Adorno, 1981)-, es decir, homogenización de los sujetos, devenido en la imperceptible imposición de un sujeto educativo cosificado a singulares formas de objetualización -empresa propia del esencialismo-. En contraste, la concepción de tiempo heterocrónico asume una praxis y un sujeto multi-temporalizado, cuyas coordenadas de enseñanza trabajan simultáneamente tantas cronologías como sea posible. La escuela es un espacio de convivencia de múltiples tiempos y espacialidades.

El problema del tiempo escolar se encuentra profundamente arraigado en las articulaciones de la gramática escolar y en la mecánica de las estructuras de escolarización. Dos puntos medulares sin mayor comprensión analítico-metodológica. La heterocronía es coherente con la singularidad y con el potencial de la multiplicidad. La presencia de la metáfora de múltiples cronologías de aprendizaje reafirma el principio epistémico-pedagógico de totalidad concebida como múltiples singularidades, fomentando una relación heurística en la que "nos encontramos multitemporalizados: viviendo simultáneamente, ritmos diferentes en un mismo tramo de tiempo" (Bal, 2018, p.10).

La concepción de tiempo y de espacio pedagógico que reclama la educación inclusiva, asumen implícitamente una noción de sensibilidad que atraviesa relacionalmente ambas categorías. Para Didi-Huberman (2017) la sensibilidad es aquello que define la experiencia. De ninguna manera, trabaja a favor de una práctica pedagógica y temporal inmanente, de sensibilidad reduccionista, tampoco asume una jerarquía teórica artificial como la impuesta por la estructura de conocimiento falsificada y mixta de inclusión. El tiempo en la experiencia escolar y en el texto didáctico de la inclusión es "puesto en ritmos por los mismos movimientos recíprocos" (p.2), una operación compleja producto de sus fuerzas de singularización reconoce la presencia de "tiempos múltiples y heterogéneos. Del mismo modo que el sujeto que se ve a sí mismo no dejaba de experimentar nuevas posturas o puntos de vista" (Didi-Huberman, 2017, p.4-5).

Finalmente, la construcción de heterocronías de aprendizaje y una espacialidad pedagógica otra o heterotópica, inaugura una crítica política de la sospecha sobre lo heurístico y lo político del contextualismo epistemológico de la educación inclusiva, esto es, destraba el falso intento de singularizar lo que siempre ratifico dicha naturaleza. Este propósito, reside el centro crítico que define la naturaleza de toda acción pedagógica. La inclusión es también, una crítica política 
de la sospecha, pues intenta devolver o imponer algo que constituye la esencia del quehacer pedagógico. Es una gramática en construcción.

\section{Conclusiones}

\section{Construir de justicia educativa: luchar por el ethos de la redistribución}

Una de las interrogantes que compone la paleta de intereses investigativos de la educación inclusiva emerge a través del estudio de los mecanismos de construcción y aseguramiento de justicia a través de la enseñanza, del currículo y de la evaluación. Este es uno de los objetivos de formación que aborda el Programa Internacional de Diplomado en Neurodiversidad y neurodidáctica: herramientas para la gestión del currículo y la evaluación a través del neuroaprendizaje, impartido por la Escuela Internacional de Postgrado del Centro de Estudios Latinoamericanos de Educación Inclusiva (CELEI).

La cristalización de modalidades de justicia educativa ratifica una compleja política de la diferencia, cuyo umbral analítico atiende a la producción de frenos al auto-desarrollo presentes en las tramas de escolarización. Coincidiendo con Young (2002), una concepción de justicia debe comenzar por un examen analítico-metodológico crítico sobre las nociones de dominación y opresión, atendiendo a las diferencias de cada grupo social, clave en la estructuración de singulares modos de relacionamiento. Un primer cuestionamiento, interroga el concepto de grupo social -punto espinoso en la interioridad de los debates de la filosofía analítica- devenido en una ontología social que rechaza la diferencia, y, en caso de aceptarla, adopta un conjunto de esencialismosindividualismos que transfieren una carga negativa sobre el sujeto, su identidad, historia e inscripción social, política y cultural. En razón de este argumento, es que la política de producción del conocimiento predominante sobre educación inclusiva trabaja a favor de un ideal de diversidad concebido en términos de asimilación.

La justicia educativa requiere de la comprensión de formas en que se constituyen las diferencias eliminando la diversidad de mecanismos de opresión -frenos al autordesarrollo- y dominación frenos a la autodeterminación- presentes en la escolarización. Siempre la pregunta por la justicia es atemporal como sostendrá Young (2002). La figuración imaginaria de justicia a la que adscribe la educación inclusiva toma distancia de un marco normativo que apela a la construcción universal y moral de derechos. Asume una concepción de justicia compleja que escucha atentamente un conjunto de demandas que serán concretizadas a través de la escolarización mediante sus tres principales dominios de construcción: lo curricular, lo didáctico y lo evaluativo. Jamás, estas dimensiones son neutras a las articulaciones de las reglas de funcionamiento de la sociedad permeadas por diversas expresiones del poder.

Una concepción de justicia compleja toma distancia de las premisas universalistas que tienden a asegurar una política normativa fundada, tal como indica, Lazarrato (2006), mediante la lógica de los todos colectivos, es decir, una perspectiva universalista y homogeneizadora que se conforma con dar a todos lo mismo -lógica de la universalidad del derecho a la educación implantada en todas las agendas gubernamentales a nivel mundial-. 
Su carácter complejo asume una concepción de justicia articulada sobre la metáfora de los todos redistributivos propuesta por Lazzarato (2006). Para el filósofo italiano esta explicación supone asegurar un piso amplio que cubra las necesidades de la totalidad de la población, aceptando que las necesidades de las personas desbordan los marcos normativos existentes y asumen que las personas no están por sobre las instituciones. Su acción trabaja a favor de la operacionalización del derecho a la educación acorde a las demandas de cada comunidad de práctica. Esta articulación política acontece en la lógica del universo-mosaico garantizando la ejecución del derecho acorde a la singularidad de cada colectividad.

Si bien, la recognición del significante de la justicia transpone las articulaciones de la política pública y sus formas de ejecución, evitando caer en sus reduccionismos políticos y heurísticos. Toda forma de justicia es una construcción histórica, esta, responderá a un conjunto de especificidades contextuales y micro-relacionales coherentes con el lenguaje de la singularidad, fuerza en permanente movimiento. En efecto, "las injusticias distributivas pueden contribuir a estas formas de opresión o incluso ser su consecuencia, pero ninguna de estas formas de opresión es reducible a la distribución, sino que todas implican estructuras sociales y relaciones que van más allá de la distribución" (Young, 2002, p.21). 


\section{Referencias}

Adorno, Th. (1981). Tres estudios sobre Hegel. Madrid: Taurus.

Armstrong, Th. (2012). El poder de la neurodiversidad. Buenos Aires: Paidós.

Bal, M. (2018). El tiempo que se toma. Contra Narrativas, Núm. 0, 8-21.

Blythe, T. (1998). La Enseñanza para la Comprensión. Guía del Docente. Buenos Aires: Paidós.

Deleuze, G. (1968). Diferencia y repetición. Barcelona: Anagrama.

Didi-Huberman, G. (2017). La imagen y las signaturas de lo político. Revista de teoría y cultura. Núm. 82. https:// mxfractal.org/articulos/RevistaFractal82Didi-huberman.php

Jiménez, C. (2000). Cerebro creativo y lúdico: hacia la construcción de una nueva didáctica para el siglo XXI. Bogotá: Cooperativa Editorial Magisterio.

Lazzarato, M. (2006). Por una política menor. Acontecimiento y política en las sociedades de control. Madrid: Traficantes de Sueños.

Massey, D. (1994). Space, place and gender. Minneapolis: University of Minnesota Press.

Núñez, A. (2010). Gilles Deleuze. La ontología menor: de la política a la estética. Revista de Estudios Sociales, $35,41.52$.

Ocampo, A. (2018). La formación del profesorado y la comprensión epistemológica de la educación inclusiva: tensiones, permeabilidades y contingencias. Santiago: Fondo Editorial CELEI.

Ocampo, A. (2020). La inclusión como proyecto de conocimiento en resistencia. Revista Espacio I+D. UNACH, 20 pp. En revisión.

Perkins, D. (2003). El aprendizaje pleno. Principios de la enseñanza para transformar la educación. Barcelona: Gedisa.

Perkins, D. (2006). La Escuela Inteligente. Del Adiestramiento de la Memoria a la Educación de la Mente. Barcelona: Gedisa.

Prigogine, Y. (1999). Espejo y reflejo. Barcelona: Gedisa.

Spivak, G. (2017). Una educación estética en la era de la globalización. México: FCE.

Stone Wiske, M. (2008). “¿Qué es la comprensión?”, en: Stone Wiske, M. (comp.). La Enseñanza para la Com- 
prensión: vinculación entre la investigación y la práctica. Buenos Aires: Paidós.

Talanquer, V. (2010). ¿Cómo piensan nuestros alumnos? Conferencia presentada en el Primer Congreso Interdisciplinario de Investigación en Educación.

Tishman, S., Palmer, P. (2005). Pensamiento Visible. http://vidarte.weebly.com/uploads/5/1/5/4/5154246/pensamiento visible.pdf

Terigi, F. (2004). El saber pedagógico frente a la crisis de la monocronía. https://lacalorconsaco.files.wordpress. com/2014/08/terigi-el-saber-pedagogico-frente2.pdf

Klenowski, V. (2004). Desarrollo del portafolios para el aprendizaje y la evaluación: procesos y principios (Vol. 98). Narcea Ediciones.

Young, I.M. (2002). Justicia y Política de la Diferencia. Valencia: Cátedra. 


\section{Copyright (c) 2020 Aldo Ocampo González}

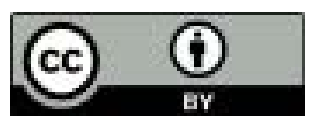

Este texto está protegido bajo una licencia internacional Creative Commons 4.0.

Usted es libre para Compartir-copiar y redistribuir el material en cualquier medio o formato - y Adaptar el documento - remezclar, transformar y crear a partir del material-para cualquier propósito, incluso para fines comerciales, siempre que cumpla las condiciones de Atribución. Usted debe dar crédito a la obra original de manera adecuada, proporcionar un enlace a la licencia, e indicar si se han realizado cambios. Puede hacerlo en cualquier forma razonable, pero no de forma tal que sugiera que tiene el apoyo del licenciante o lo recibe por el uso que hace de la obra.

\section{$\underline{\text { Resumen de licencia - Texto completo de la licencia }}$}

